\section{On the homogeneity of the affine connection model for mechanical control systems*}

Francesco Bullo ${ }^{\dagger} \quad$ Andrew D. Lewis ${ }^{\ddagger}$

$29 / 02 / 2000$

\section{Abstract}

This work presents a review of a number of control results for mechanical systems. The key technical results derive mainly from the homogeneity properties of affine connection models for a large class of mechanical systems. Recent results on nonlinear controllability and on series expansions are presented in a unified fashion.

Keywords. mechanical control systems, affine connections, homogeneity

AMS Subject Classifications. 53B05, 70H03, 70Q05, 93B05, 93B29

\section{Introduction}

In this paper we provide a review of some recent work concerning the employment of an affine connection framework to study mechanical systems. The emphasis of the presentation here is on the homogeneity properties possessed by these systems, and how this arises in various results, especially those of the authors. It is this property of homogeneity which accounts for a great deal of the structure seen in so-called affine connection control systems. The structure of these systems makes them an ideal proving ground for many techniques in nonlinear control - the systems are simple enough that one may fruitfully approach difficult problems, but are nontrivial enough to require sophisticated machinery to have any degree of success. For example, typical linearization techniques are not useful in this category of control systems.

The classic structure of mechanical system exploited in stabilization problems is passivity. Indeed, numerous important control problems rely in their essence on the existence of a total energy function and its use as a candidate Lyapunov function, see for example the books [Arimoto 1996, Ortega, Loria, Nicklasson, and Sira-Ramirez 1998]. This paper focuses on a different property of mechanical systems, that is, their homogeneity. This property characterizes the Lie algebraic structure of mechanical systems, and accordingly, it plays a key rôle in nonlinear controllability, normal forms, series expansions, averaging, *To appear in the Proceedings of the 39 th IEEE CDC

'Research Assistant Professor, COORDINATED SCIENCE LABoratory, University of Illinois, URbanaChampaign, Urbana-Champaign, IL 61801, U.S.A.

Email: bullo@uiuc. edu, URL: http://motion.csl.uiuc. edu/ bullo/

Research supported in part by NSF grant CMS-0100162.

${ }^{\ddagger}$ Assistant Professor, Department of Mathematics and Statistics, Queen’s University, Kingston, ON K7L 3N6, CANADA

Email: andrew@mast.queensu.ca, URL: http://www.mast.queensu.ca/ andrew/

Research supported in part by a grant from the Natural Sciences and Engineering Research Council of Canada. optimal control and various other areas of control theory. It is a contention of this paper that this property has for long time been neglected, and that instead its consequences should be investigated with increased energy.

This paper relies on the notion of affine connection control systems to model a large class of systems which are of current interest in the control community. Broadly speaking, Lagrangian mechanical systems with kinetic energy Lagrangians are effectively modeled in the affine connection framework, and this is the topic of the papers [Lewis and Murray 1997a] and [Bullo, Leonard, and Lewis 2000]. If one adds constraints linear in velocity to this class of systems, the resulting systems may still be modeled using affine connections, and the control setting for this is described, for example, by Lewis [Lewis 2000b]. This class of Lagrangian control systems may be subjected to the following categorization which is not mathematically complete, but which identifies the special structure present in certain application areas:

1. simple systems with integrable forces (robotic manipulators and other systems with Lagrangian equal to kinetic energy, and forces applied in coordinate directions);

2. invariant systems on Lie groups (aerospace and underwater vehicles and other systems with a matrix group as configuration space, with body fixed forces, and with invariant kinetic energy);

3. systems subject to nonholonomic constraints (mobile robots and locomotion devices with drift, e.g., snakeboard, roller racer, and $G$-snakes).

Among the research problems addressed by the authors and others in the area of affine connection control systems are local nonlinear controllability [Lewis and Murray 1997a], normal forms [Lewis 1999, Lewis and Murray 1997b], series expansions [Bullo 2001] and algorithms for motion planning [Bullo, Leonard, and Lewis 2000], averaging via the average potential [Baillieul 1993, Bullo 2002], and optimal control [Lewis 2000a, Sontag and Sussmann 1986]. Below we touch on some of these areas, and address how the property of homogeneity plays an important rôle. Specifically, we review some of the existing work on controllability and series expansions. The importance of these results is established by their use in generating motion planning algorithms [Bullo, Leonard, and Lewis 2000, Ostrowski 2000, Zhang and Ostrowski 2000].

\section{Affine connections and mechanics}

In this section we begin with a brief overview of affine connections, and how they come up in mechanics. We also mention how homogeneity enters the picture in terms of the basic problem data for the control problem.

2.1. Affine connections. We refer to [Kobayashi and Nomizu 1963] for a comprehensive treatment on affine connections and Riemannian geometry. An affine connection on a manifold $Q$ assigns to a pair of vector fields $X, Y$ a vector field $\nabla_{X} Y$ such that for any function $f$ and for any third vector field $Z$ :

1. $\nabla_{f X+Y} Z=f \nabla_{X} Z+\nabla_{Y} Z$, 
2. $\nabla_{X}(f Y+Z)=\left(\mathscr{L}_{X} f\right) Y+f \nabla_{X} Y+\nabla_{X} Z$.

We also say that $\nabla_{X} Y$ is the covariant derivative of $Y$ with respect to $X$. Vector fields can also be covariantly differentiated along curves, and this concept is employed in writing the Euler-Lagrange equations. Consider a differentiable curve $\gamma:[0,1] \rightarrow Q$ and a vector field along $\gamma$, that is, a map $v:[0,1] \rightarrow T Q$ such that $\pi(v(t))=\gamma(t)$ for all $t \in[0,1]$. Let the vector field $V$ satisfy $V(\gamma(t))=v(t)$. The covariant derivative of the vector field $v$ along $\gamma$ is defined by

$$
\frac{D v(t)}{d t} \triangleq \nabla_{\dot{\gamma}(t)} v(t)=\left.\nabla_{\dot{\gamma}(t)} V(q)\right|_{q=\gamma(t)} .
$$

In a system of local coordinates $\left(q^{1}, \ldots, q^{n}\right)$, an affine connection is uniquely determined by its Christoffel symbols $\Gamma_{i j}^{i}$

$$
\nabla_{\frac{\partial}{\partial q^{i}}}\left(\frac{\partial}{\partial q^{j}}\right)=\Gamma_{i j}^{k} \frac{\partial}{\partial q^{k}}
$$

and accordingly, the covariant derivative of a vector field is written as

$$
\nabla_{X} Y=\left(\frac{\partial Y^{i}}{\partial q^{j}} X^{j}+\Gamma_{j k}^{i} X^{j} Y^{k}\right) \frac{\partial}{\partial q^{i}} .
$$

In settings where $Q$ possesses a Riemannian metric $g$ (such as is provided, for example, by kinetic energy), one derives a canonical affine connection associated with $g$. This connection is called the Levi-Civita affine connection, and is most directly characterized by its Christoffel symbols, which are given in terms of the metric components as follows:

$$
\Gamma_{j k}^{i}=\frac{1}{2} g^{i \ell}\left(\frac{\partial g_{j \ell}}{\partial q^{k}}+\frac{\partial g_{k \ell}}{\partial q^{j}}-\frac{\partial g_{j k}}{\partial q^{\ell}}\right) .
$$

Although this is the affine connection commonly seen in applications, our treatment here is not restricted to the Levi-Civita connection. Indeed, one of the remarkable features of affine connection control systems is that many of the results apply to systems with arbitrary affine connections. It is this fact which makes easy the adaptation of the results to systems with nonholonomic constraints.

2.2. Control systems described by affine connections. We introduce a class of control systems that is a generalization of Lagrangian control systems. As an approach to modeling of vehicles and robotic manipulators, for example, this is common to a number of recent papers; see [Bloch and Crouch 1995, Bullo 2001, Lewis 2000b, Lewis and Murray 1997a]. An affine connection control system is defined by the following objects:

1. an $n$-dimensional configuration manifold $Q$, with $q \in Q$ being the configuration of the system and $v_{q} \in T_{q} Q$ being the system's velocity,

2. an affine connection $\nabla$ on $Q$, whose Christoffel symbols are $\left\{\Gamma_{j k}^{i}: i, j, k=1, \ldots, n\right\}$,

3. a family of input vector fields $\mathscr{Y}=\left\{Y_{1}, \ldots, Y_{m}\right\}$ on $Q$.
The corresponding equations of motion are written as

$$
\nabla_{\dot{q}(t)} \dot{q}(t)=u^{a}(t) Y_{a}(q(t))
$$

or equivalently in coordinates as

$$
\ddot{q}^{i}+\Gamma_{j k}^{i}(q) \dot{q}^{j} \dot{q}^{k}=u^{a}(t) Y_{a}^{i}(q),
$$

where the indices $i, j, k \in\{1, \ldots, n\}$. These equations are a generalized form of the EulerLagrange equations. That is to say, if one takes for $\nabla$ the Levi-Civita affine connection associated with a kinetic energy Riemannian metric $g$, then the equations (2.1) are the forced Euler Lagrange equations for the associated kinetic energy Lagrangian and with input forces modeled by the vector fields $Y_{1}, \ldots, Y_{m}$. However, as we mentioned in the previous section, we do not wish to restrict our attention to Levi-Civita affine connections, and so the equations (2.1) in consequence give, for example, the forced equations of motion for a nonholonomic system with a kinetic energy Lagrangian, and constraints linear in velocity.

The second-order system in equation $(2.2)$ can be written as a first-order differential equation on the tangent bundle $T Q$. Using $\left\{\frac{\partial}{\partial q^{i}}, \frac{\partial}{\partial \nu^{i}}\right\}_{1<i<n}$ as a basis for vector fields on the tangent bundle to $T Q$, we define vector fields $Z$ and $Y_{a}^{\text {lift }}, a=1, \ldots, m$, on $T Q$ by

$$
\begin{gathered}
Z\left(v_{q}\right)=v^{i} \frac{\partial}{\partial q^{i}}-\Gamma(q)_{j k}^{i} v^{j} v^{k} \frac{\partial}{\partial v^{i}}, \\
Y_{a}^{\text {lift }}\left(v_{q}\right)=Y_{a}^{i}(q) \frac{\partial}{\partial v^{i}},
\end{gathered}
$$

so that the control system is rewritten as

$$
\dot{v}(t)=Z(v(t))+u^{a}(t) Y_{a}^{\text {lift }}(v(t)),
$$

where $t \mapsto v(t)$ is now a curve in $T Q$ describing the evolution of a first-order control affine system. We refer to [Lang 1995, Lewis and Murray 1997a] for coordinate independent definitions of the lifting operation $Y_{a} \rightarrow Y_{a}^{\text {lift }}$ and of the drift vector field $Z$. The latter vector field is called the geodesic spray.

2.3. Homogeneity and Lie algebraic structure. A fundamental observation about the structure of the control system (2.1) is the polynomial dependence of the vector fields $Z$ and $Y^{\text {lift }}$ on the velocity variables $v^{i}$. This structure leads to some enormous simplifications when performing iterated Lie brackets between the vector fields in the set $\left\{Z, Y_{1}^{\text {lift }}, \ldots, Y_{m}^{\text {lift }}\right\}$. Apart from the papers of the authors concerning the consequences of the structure of these Lie brackets, we refer to the work of Sontag and Sussmann [Sontag and Sussmann 1986] on time-optimal control for robotic manipulators. Other work on optimal control for affine connection control systems is that of Lewis [Lewis 2000a]

We focus here on the notion of geometric homogeneity ${ }^{1}$ as described in [Kawski 1995]. Generally, given two vector fields $X$ and $X_{E}$, we say that the vector field $X$ is homogeneous with degree $m \in \mathbb{Z}$ with respect to $X_{E}$ if

$$
\left[X_{E}, X\right]=m X .
$$

${ }^{1}$ Geometric homogeneity corresponds to the existence of an infinitesimal symmetry in the equations of motion. For control systems described by an affine connection the symmetry is invariance under affine time-scaling transformations. 
For affine connection control systems, we introduce the Liouville vector field $L$ on $T Q$ (see [Libermann and Marle 1987, page 64]), as

$$
L=v^{i} \frac{\partial}{\partial v^{i}} .
$$

Straightforward computations verify the following statements.

2.1 Lemma: Let $\nabla$ be an affine connection on $Q$ with geodesic spray $Z$, and let $Y$ be a vector field on $Q$. The following statements hold:

$$
\text { 1. }[L, Z]=(+1) Z \text {; }
$$$$
\text { 2. }\left[L, Y^{\mathrm{lift}}\right]=(-1) Y^{\mathrm{lift}} .
$$

Hence, the vector field $Z$ is homogeneous of degree +1 , and the vector field $Y^{\text {lift }}$ is homogeneous of degree -1 with respect to the Liouville vector field. In the following, a vector field $X$ on $T Q$ is simply homogeneous of degree $\boldsymbol{m} \in \mathbb{Z}$ if it is homogeneous of degree $m$ with respect to $L$. Let $\mathcal{P}_{j}$ be the set of vector fields on $T Q$ of homogeneous degree $j$, so that

$$
Z \in \mathcal{P}_{1}, \quad \text { and } \quad Y^{\mathrm{lift}} \in \mathcal{P}_{-1} .
$$

Let us leave our general discussion of homogeneity at that for the moment, and in the next section we will investigate these properties further, and illustrate how they may be used in an investigation of nonlinear controllability for affine connection control systems.

\section{Controllability of affine connection control systems}

The matter of controllability for affine connection control systems was first undertaken systematically by Lewis and Murray [Lewis and Murray 1997a]. Here the precise character of the Lie bracket structure for affine connection control systems was undertaken, and in the presence of a potential energy term in the Lagrangian. In this section, we distill the essence of this structure without potential energy (with potential energy, the systems are not affine connection control systems as we have defined them in Section 2.2). As we shall see, the resulting structure allows us to quickly understand the character of the set of configurations one can reach starting from a state with zero velocity.

First we observe that the sets $\mathcal{P}_{j}$ enjoy various interesting properties: Figure 1 illustrates them, and their proof is via direct computation. Here are the properties illustrated in the table, but expressed via formulas:

1. $\left[\mathcal{P}_{i}, \mathcal{P}_{j}\right] \subset \mathcal{P}_{i+j}$. that is, the Lie bracket between a vector field in $\mathcal{P}_{i}$ and a vector field in $\mathcal{P}_{j}$ belongs to $\mathcal{P}_{i+j}$;

2. $\mathcal{P}_{k}=\emptyset$ for all $k \leq-2$;

3. for all $X\left(v_{q}\right) \in \mathcal{P}_{k}$ with $k \geq 1, X\left(0_{q}\right)=0_{q}$.

Here $0_{q}$ is the zero vector in the tangent space $T_{q} Q$. The key observation here is that all brackets are homogeneous of some degree, and if one is evaluating brackets at points of zero velocity, the only contributions will come from those brackets which are homogeneous

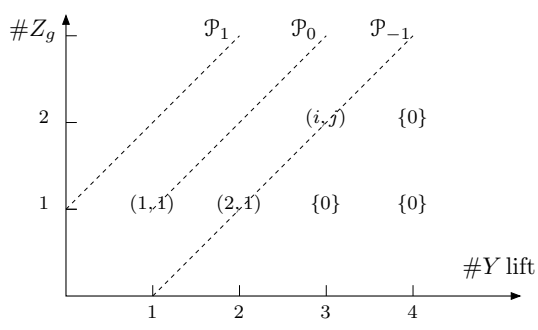

FigURE 1. Table of Lie brackets between the drift vector field $Z$ and the input vector fields $Y_{a}^{\text {litt }}$. The $(i, j)$ th position contains Lie brackets with $i$ copies of $Y^{\text {lift }}$ and $j$ copies of $Z$. The corresponding homogeneous degree is $j-i$. All Lie brackets to the right of $\mathcal{P}_{-1}$ exactly vanish. All Lie brackets to the left of $\mathcal{P}_{-1}$ vanish when evaluated at $v_{q}=0_{q}$.

of degree -1 or 0 . It turns out that one can easily characterize these brackets, and this is exactly what is undertaken by Lewis and Murray [Lewis and Murray 1997a].

To understand what a vector field from $\mathcal{P}_{i}$ looks like, let us work in local coordinates. We write a vector field $X$ on $T Q$ as

$$
X=X_{h}^{i} \frac{\partial}{\partial q^{i}}+X_{v}^{i} \frac{\partial}{\partial v^{i}} .
$$

Here we think of the components $X_{h}^{i}, i=1, \ldots, n$, as being "horizontal" and the components $X_{v}^{i}, i=1, \ldots, n$, as being "vertical." Let $\mathcal{H}_{i}$ be the set of scalar functions in the local chart for $T Q$ which are arbitrary functions of $q$ and which are homogeneous polynomials in $\left\{v^{1}, \ldots, v^{n}\right\}$ of degree $i$. One verifies that a vector field $X$ on $T Q$ of the form (3.1) is in $\mathcal{P}_{i}$ exactly when the functions $X_{h}^{i}, i=1, \ldots, n$, are in $\mathcal{H}_{i}$, and the functions $X_{v}^{i}, i=1, \ldots, n$, are in $\mathcal{H}_{i+1}$.

Let us focus for the moment on the Lie bracket $\left[Y_{a}^{\text {lift }},\left[Z, Y_{b}^{\text {lift }}\right]\right]$ where $a, b \in\{1, \ldots, m\}$. Since this Lie bracket belongs to $\mathcal{P}_{-1}$, there must exist a vector field on $Q$, which we denote $\left\langle Y_{a}: Y_{b}\right\rangle$, such that

$$
\left\langle Y_{a}: Y_{b}\right\rangle^{\text {lift }}=\left[Y_{b}^{\text {lift }},\left[Z, Y_{a}^{\text {lift }}\right]\right] .
$$

This vector field we call the symmetric product between $Y_{a}$ and $Y_{b}$ and a direct computation shows that it satisfies

$$
\left\langle Y_{b}: Y_{a}\right\rangle=\nabla_{Y_{a}} Y_{b}+\nabla_{Y_{b}} Y_{a},
$$

or equivalently in coordinates

$$
\left\langle Y_{b}: Y_{a}\right\rangle^{i}=\frac{\partial Y_{a}^{i}}{\partial q^{j}} Y_{b}^{j}+\frac{\partial Y_{b}^{i}}{\partial q^{j}} Y_{a}^{j}+\Gamma_{j k}^{i}\left(Y_{a}^{j} Y_{b}^{k}+Y_{a}^{k} Y_{b}^{j}\right) .
$$


The adjective "symmetric" comes from the obvious equality $\left\langle Y_{a}: Y_{b}\right\rangle=\left\langle Y_{b}: Y_{a}\right\rangle$. Without reference to the formula (3.2), symmetry can be shown to follow from the Jacobi identity and the fact that $\left[Y_{\mathrm{a} t}^{\text {lift }}, Y_{b}^{\text {lift }}\right]=0$. It turns out, in fact, that all Lie brackets of vector fields from the set $\left\{Z, Y_{\text {lift }} \ldots, Y_{\text {lift }}\right\}$ which are also vector fields in $\mathcal{P}_{-1}$ are vertical lifts of iterated symmetric products of the vector fields $\left\{Y_{1}, \ldots, Y_{m}\right\}$. We denote the distribution spanned by all such iterated symmetric products by $\overline{\operatorname{Sym}}(\mathscr{Y})$.

Now let us focus on another type of bracket, those of the form $\left[\left[Z, Y_{a}^{\text {lift }}\right],\left[Z, Y_{b}^{\text {lift }}\right]\right]$ for $a, b \in\{1, \ldots, m\}$. This bracket, under our classification scheme, is in $\mathcal{P}_{0}$. Therefore, if $\tau_{Q}: T Q \rightarrow Q$ is the tangent bundle projection, there is a vector field $X_{a b}$ on $Q$ which satisfies

$$
\tau_{Q}\left(\left[\left[Z, Y_{a}^{\mathrm{lift}}\right],\left[Z, Y_{b}^{\mathrm{lift}}\right]\right]\left(v_{q}\right)\right)=X_{a b}(q) .
$$

That is, the vector field $\left[\left[Z, Y_{a}^{\text {lift }}\right],\left[Z, Y_{b}^{\text {lift }}\right]\right]$ is projectable with respect to the tangent bundle projection. A routine computation shows that in fact $X_{a b}=-\left[Y_{a}, Y_{b}\right]$. Thus when we evaluate brackets in $\mathcal{P}_{0}$, we expect to get something involving Lie brackets of vector fields whose vertical lifts are brackets from $\mathcal{P}_{-1}$. Indeed, all Lie brackets of vector fields from the set $\left\{Z, Y_{1}^{\text {lift }}, \ldots, Y_{m}^{\text {lift }}\right\}$ which are also vector fields in $\mathcal{P}_{0}$ project to a vector field on $Q$ which is a Lie bracket of two iterated symmetric products. Somewhat more precisely, if $\overline{\operatorname{Lie}}(D)$ denotes the smallest integrable distribution containing a distribution $D$, the distribution on $Q$ generated by the projection to $Q$ of brackets from $\mathcal{P}_{0}$ is given by $\overline{\operatorname{Lie}}(\overline{\operatorname{Sym}}(\mathscr{Y}))$.

To summarize, we have made believable the following result of Lewis and Murray [Lewis and Murray 1997a].

3.1 THEOREM: For an analytic affine connection control system, the set of configurations reachable from $q \in Q$ starting at zero initial velocity forms an open subset of the integral manifold through $q$ of the integrable distribution $\overline{\operatorname{Lie}}(\overline{\operatorname{Sym}}(\mathscr{Y}))$.

In particular, if $\overline{\operatorname{Lie}}(\overline{\operatorname{Sym}}(\mathscr{Y}))$ has full rank at $q \in Q$, the set of configurations reachable from initial condition $0_{q}$ contains a nonempty open subset of $Q$.

To make this precise requires some effort, but we hope to have made it clear the important rôle that homogeneity plays in understanding the special structure of affine connection control systems.

\section{A series expansion for the forced evolution from rest}

As in the previous section, the homogeneity and Lie algebraic structure of mechanical systems leads to a novel and instructive characterization of their flow. Assuming zero initial velocity, the evolution of the second-order initial value problem in equation (2.3) can be described via a first-order differential equation. Precise statements and proof are available in [Bullo 2001]; a preliminary version of these results appeared in [Bullo 1999]. In this section since the results are local, we let $Q$ be an open subset of $\mathbb{R}^{n}$.

In the computation of series expansions as well as in the general study of perturbation methods for differential equations, one key tool is the variation of constants formula. We start by introducing some notation. A time-varying vector field on $Q,(q, t) \mapsto X(q, t)$, gives rise to the initial value problem on $Q$

$$
\dot{q}(t)=X(q, t), \quad q(0)=q_{0} .
$$

We denote its solution at time $T$ by $q(T)=\Phi_{0, T}^{X}\left(q_{0}\right)$, thus defining the flow $T \mapsto \Phi_{0, T}^{X}$. Consider the initial value problem

$$
\dot{q}(t)=X(q, t)+Y(q, t), \quad q(0)=q_{0},
$$

where $X$ and $Y$ are analytic time-varying vector fields. If we regard $X$ as a perturbation to the vector field $Y$, we can describe the flow of $X+Y$ in terms of a nominal and perturbed flow. For a diffeomorphism $\phi: Q \rightarrow Q$ and a vector field $X$ on $Q, \phi^{*} X$ denotes the pull-back of $X$ by $\phi$. With this notation, the following relationship is referred to as the variation of constants formula and describes the perturbed flow:

$$
\Phi_{0, t}^{X+Y}=\Phi_{0, t}^{Y} \circ \Phi_{0, t}^{\left(\Phi_{0, t}^{Y}\right)^{*} X} .
$$

The result is proven in [Agrachev and Gamkrelidze 1978, equation (3.15)], see also [Bullo 2001, Appendix A.1].

If $X$ and $Y$ are time-invariant, the classic infinitesimal Campbell-Backer-Hausdorff formula provides a means of computing the pull-back:

$$
\left(\Phi_{0, t}^{Y}\right)^{*} X=\sum_{k=0}^{\infty} \operatorname{ad}_{Y}^{k} X \frac{t^{k}}{k !} .
$$

If instead $X$ and $Y$ are time-varying, a generalized expression is given by Agrachev and Gamkrelidze [1978]:

$$
\left(\Phi_{0, t}^{Y}\right)^{*} X(q, t)=X(q, t)+\sum_{k=1}^{\infty} \int_{0}^{t} \cdots \int_{0}^{s_{k-1}}
$$

$$
\left(\operatorname{ad}_{Y\left(q, s_{k}\right)} \ldots \operatorname{ad}_{Y\left(q, s_{1}\right)} X(q, t)\right) \mathrm{d} s_{k} \ldots \mathrm{d} s_{1} .
$$

Although the convergence of this series expansion is a delicate manner, the following straightforward statement suffices in our analysis: if all the Lie brackets $\operatorname{ad}_{Y\left(s_{k}\right)} \ldots \operatorname{ad}_{Y\left(s_{1}\right)} X$ vanish for all $k$ greater than some finite integer, then the series in equation (4.3) becomes a finite sum.

Next, we apply this result to the differential equation (2.3) on $T Q$, which we rewrite here for convenience:

$$
\dot{v}=Z(v)+Y(v, t)^{\text {lift }}
$$

where $Y(v, t)=u^{a}(t) Y_{a}^{\text {lift }}(v)$. The homogeneous structure described in Figure 1 can be used to simplify the application of the variation of constants formula. Let the geodesic spray $Z$ play the role of the perturbation to the vector field $Y^{\text {lift }}$. Then the infinite series in equation (4.3) collapses.

Let us briefly describe this simplification. The solution to the equation (4.4) from the zero velocity state $0_{q_{0}}$ is

$$
v(T)=\Phi_{0, T}^{Z+Y^{\text {lift }}}\left(0_{q_{0}}\right) .
$$

Utilizing equation (4.2) we compute

$$
v(T)=\Phi_{0, T}^{Y^{l i f t}}(w(T))=w(T)+\int_{0}^{t} Y(q, s) \mathrm{d} s
$$


where some straightforward manipulations lead to

$$
\begin{aligned}
\dot{w}(t)= & \left(\Phi_{0, T}^{Y^{\text {lift }}}\right)^{*} Z(w(t)) \\
= & Z+\int_{0}^{t}\left[Y^{\text {lift }}(q, s) Z\right] \mathrm{d} s- \\
& \frac{1}{2} \int_{0}^{t} \int_{0}^{t}\left\langle Y\left(q, s_{1}\right): Y\left(q, s_{2}\right)\right\rangle^{\text {lift }} \mathrm{d} s_{1} \mathrm{~d} s_{2},
\end{aligned}
$$

with initial condition $w(0)=0_{q_{0}}$.

The key observation comes now: the transformed initial value problem in $w$ enjoys the same homogeneity properties as the original one in equation (2.3). In other words, the resulting system satisfies a set of equations similar to the original one, except for some different forcing terms. One can therefore infinitely iterate this procedure and under mild assumptions obtain a locally convergent solution. Making this statements precise is the purpose of [Bullo 2001]. We here summarize the result as follows.

4.1 Theorem: Define recursively the time-varying vector fields $V_{k}$ :

$$
\begin{aligned}
& V_{1}(q, t)=\int_{0}^{t} u^{a}(s) Y_{a}(q) \mathrm{d} s \\
& V_{k}(q, t)=-\frac{1}{2} \sum_{j=1}^{k-1} \int_{0}^{t}\left\langle V_{j}(q, s): V_{k-j}(q, s)\right\rangle \mathrm{d} s .
\end{aligned}
$$

The solution $t \rightarrow q(t)$ to equation (2.1) satisfies the formal series expansion

$$
\dot{q}(t)=\sum_{k=1}^{\infty} V_{k}(q(t), t)
$$

We refer to [Bullo 2001] for a detailed convergence analysis of the series: local absolute and uniform convergence can be proven under the assumption of analyticity and boundedness of the various quantities involved.

\section{Simplifications in example systems}

While the treatment presented up to here is always applicable, there are two situations in which further structure in the affine connection $\nabla$ and in the input forces $Y_{a}$ simplifies the computation of symmetric products. In this section we illustrate how these simplifications spill over to our series solution results of Section 4. Similar simplifications hold, of course, for the controllability results of Section 3 .

5.1. Simple systems with integrable forces. In this section we let $Q$ be an open subset of $\mathbb{R}^{n}$.

Here we consider systems with kinetic energy Lagrangians; if one allows potential forces, such systems are referred to as "simple." Regarding inputs, we make the assumption that the forces for the system span an integrable codistribution. The affine connection of a simple system is the Levi-Civita connection associated with the kinetic energy Riemannian metric $\langle\cdot, \cdot\rangle$. If the control forces are integrable, then we may choose the input vector fields $Y_{a}$ so that there locally exists a scalar function $\varphi_{a}$ such that

$$
Y_{a}(q)=\operatorname{grad} \varphi_{a}(q),
$$

where grad is taken with respect to the kinetic energy Riemannian metric.

One remarkable simplification takes place under these assumptions: the set of gradient vector fields is closed under the operation of symmetric product. Let $\varphi_{1}, \varphi_{2}$ be scalar functions on $\mathbb{R}^{n}$ and define a symmetric product between functions according to

$$
\left\langle\varphi_{1}: \varphi_{2}\right\rangle \triangleq\left\langle\operatorname{grad} \varphi_{1}, \operatorname{grad} \varphi_{2}\right\rangle .
$$

Then the symmetric product of the corresponding gradient vector fields equals the gradient of the symmetric product of the functions:

$$
\left\langle\operatorname{grad} \varphi_{1}: \operatorname{grad} \varphi_{2}\right\rangle=\operatorname{grad}\left\langle\varphi_{1}: \varphi_{2}\right\rangle .
$$

Accordingly, Theorem 4.1 can be restated as follows.

5.1 Lemma: For a simple system with integrable forces, define recursively the time-varying functions:

$$
\begin{aligned}
\phi_{1}(q, t) & =\int_{0}^{t} u^{a}(s) \varphi_{a}(q) \mathrm{d} s \\
& \vdots \\
\phi_{k}(q, t) & =\frac{1}{2} \sum_{j=1}^{k-1} \int_{0}^{t}\left\langle\phi_{j}(q, s): \phi_{k-j}(q, s)\right\rangle \mathrm{d} s, \quad k \geq 2 .
\end{aligned}
$$

Then the solution $q:[0, T] \rightarrow Q$ to (2.1) satisfies

$$
\dot{q}(t)=\operatorname{grad} \sum_{k=1}^{\infty} \phi_{k}(q(t), t)
$$

In other words, the flow of a simple system forced from rest is written as a (time-varying) gradient flow.

5.2. Invariant systems on Lie groups. In this section we briefly investigate systems with kinetic energy and input forces which are completely invariant under a certain group action. These system have a configuration space $G$ with the structure of an $n$ dimensional matrix Lie group. Systems in this class include satellites, hovercraft, and underwater vehicles.

The equations of motion (2.1) decouple into a kinematic and dynamic equation in the configuration variable $g \in G$ and the body velocity $v$ which lies in the Lie algebra $\mathfrak{g}$ of the group $G .^{2}$ The kinematic equation can be written as a matrix differential equation using matrix group notation $\dot{g}=g \widehat{v}$; we refer to [Murray, Li, and Sastry 1994] for the details. The

${ }^{2}$ For matrix Lie groups, $\mathfrak{g}$ is a subalgebra of the Lie algebra of $n \times n$ matrices with bracket given by the matrix commutator. 
dynamic equation, sometimes referred to as the Euler-Poincarè equation, can be written after a choice of basis for $\mathfrak{g}$, as

$$
\dot{v}^{i}+\gamma_{j k}^{i} v^{j} v^{k}=u^{a}(t) y_{a}^{i},
$$

where the coefficients $\gamma_{j k}^{i}$ are uniquely determined from the knowledge of the inertia metric and of the group $G$. The input vectors $y_{a}$ are constant.

Within this setting, the result in Theorem 4.1 is summarized as follows. The solution to the equation (5.4) with initial condition $v(0)=0$ is $v(t)=\sum_{k=1}^{\infty} v_{k}(t)$, where

$$
\begin{aligned}
& v_{1}(t)=\int_{0}^{t} u^{a}(a) y_{a} \mathrm{~d} s \\
& v_{k}(t)=-\frac{1}{2} \sum_{j=1}^{k-1} \int_{0}^{t}\left\langle v_{j}(s): v_{k-j}(s)\right\rangle \mathrm{d} s, \quad k \geq 2,
\end{aligned}
$$

and where the symmetric product between velocity vectors is $\langle x: y\rangle^{i}=-2 \gamma_{j k}^{i} x^{j} y^{k}$. Local convergence for the series expansion can be easily established in this setting. This result agrees and indeed supersedes the ones presented in [Bullo, Leonard, and Lewis 2000].

\section{Closing remarks}

In this paper we have explored a few of the consequences of homogeneity in the affine connection model for mechanical control systems. In particular, we have provided an interpretation in terms of homogeneity of some of the controllability results of Lewis and Murray [1997a], and of the time series results of Bullo [2001]. We see how these homogeneity properties nicely characterize much of the structured behaviour of affine connection control systems. It is precisely this structured behaviour which has made possible the recent progress in the investigation of these systems, and doubtless continued progress can be made along these lines, yielding results of fundamental and practical interest.

Acknowledgments. The research performed by the first author is supported by the Campus Research Board of the University of Illinois. The second author has enjoyed the support of a grant from the Natural Sciences and Engineering Research Council of Canada.

\section{References}

Agrachev, A. A. and Gamkrelidze, R. V. [1978] The exponential representation of flows and the chronological calculus, Mathematics of the USSR-Sbornik, 107(4), 467-532.

Arimoto, S. [1996] Control Theory of Non-linear Mechanical Systems: A Passivity-Based and Circuit-Theoretic Approach, volume 49 of OESS, Oxford University Press, Walton Street, Oxford OX2 6DP, ISBN 0-198-56291-8.

Baillieul, J. [1993] Stable average motions of mechanical systems subject to periodic forcing in Dynamics and Control of Mechanical Systems, M. J. Enos, editor, volume 1, pages 1-23, Fields Institute, ISBN 0-821-89200-2.
Bloch, A. M. and Crouch, P. E. [1995] Nonholonomic and vakonomic control systems on Riemannian manifolds, SIAM Journal on Control and Optimization, 33(1), 126-148.

Bullo, F. [1999] A series describing the evolution of mechanical control systems, in Proceedings of the IFAC World Congress, pages 479-485, IFAC, Beijing, China.

- [2001] Series expansions for the evolution of mechanical control systems, SIAM Journal on Control and Optimization, 40(1), 166-190.

- [2002] Averaging and vibrational control of mechanical systems, SIAM Journal on Control and Optimization, 40(1), 542-562.

Bullo, F., Leonard, N. E., and Lewis, A. D. [2000] Controllability and motion algorithms for underactuated Lagrangian systems on Lie groups, Institute of Electrical and Electronics Engineers. Transactions on Automatic Control, 45(8), 1437-1454.

Kawski, M. [1995] Geometric homogeneity and applications to stabilization, in Nonlinear Control Systems Design, A. Krener and D. Mayne, editors, pages 147-152, Elsevier Publishing Company, Amsterdam/London/New York

Kobayashi, S. and Nomizu, K. [1963] Foundations of Differential Geometry, Volumes I and II, number 15 in Interscience Tracts in Pure and Applied Mathematics, Interscience Publishers, New York.

Lang, S. [1995] Differential and Riemannian Manifolds, number 160 in Graduate Texts in Mathematics, Springer-Verlag, New York-Heidelberg-Berlin, ISBN 0-387-94338-2.

Lewis, A. D. [1999] When is a mechanical control system kinematic?, in Proceedings of the 38th IEEE Conference on Decision and Control, pages 1162-1167, Institute of Electrical and Electronics Engineers, Phoenix, AZ.

- [2000a] The geometry of the maximum principle for affine connection control systems, Preprint, available online at http://penelope.mast.queensu.ca/ andrew/.

- [2000b] Simple mechanical control systems with constraints, Institute of Electrical and Electronics Engineers. Transactions on Automatic Control, 45(8), 1420-1436.

Lewis, A. D. and Murray, R. M. [1997a] Controllability of simple mechanical control systems, SIAM Journal on Control and Optimization, 35(3), 766-790.

- [1997b] Decompositions of control systems on manifolds with an affine connection, Systems \& Control Letters, 31(4), 199-205.

Libermann, P. and Marle, C.-M. [1987] Symplectic Geometry and Analytical Mechanics, number 35 in Mathematics and its Applications, D. Reidel Publishing Company, Dordrecht/Boston/Lancaster/Tokyo, ISBN 90-2772-438-5.

Murray, R. M., Li, Z. X., and Sastry, S. S. [1994] A Mathematical Introduction to Robotic Manipulation, CRC Press, 2000 Corporate Blvd., N.W., Boca Raton, Florida 33431, ISBN 0-8493-7981-4 
Ortega, R., Loria, A., Nicklasson, P. J., and Sira-Ramirez, H. [1998] Passivity-Based Control of Euler-Lagrange Systems: Mechanical, Electrical and Electromechanical Applications, Communications and Control Engineering Series, Springer-Verlag, New York-HeidelbergBerlin, ISBN 1-85233-016-3.

Ostrowski, J. P. [2000] Steering for a class of dynamic nonholonomic systems, Institute of Electrical and Electronics Engineers. Transactions on Automatic Control, 45(8), 14921497.

Sontag, E. D. and Sussmann, H. J. [1986] Time-optimal control of manipulators, in Proceedings of the IEEE International Conference on Robotics and Automation, Institute of Electrical and Electronics Engineers, San Francisco, CA.

Zhang, H. and Ostrowski, J. P. [2000] Control algorithms using affine connections on principal fiber bundles, in IFAC Workshop on Lagrangian and Hamiltonian Methods for Nonlinear Control, N. E. Leonard and R. Ortega, editors, pages 134-139, IFAC, Princeton, New Jersey. 